\title{
Electrochemical Studies of Camptothecin and Its Interaction with Human Serum Albumin
}

\author{
Jing Zhao ${ }^{1}$, Xiaofeng Zheng ${ }^{1}$, Wei Xing ${ }^{1}$, Junyi Huang ${ }^{2}$ and Genxi Li ${ }^{1,2^{*}}$ \\ 1 Department of Biochemistry and National Key Laboratory of Pharmaceutical Biotechnology, Nanjing \\ University, Nanjing 210093, P. R. China
}

2 School of Life Science and Shanghai Key Laboratory of Bio-Energy Crops, Shanghai University, Shanghai 200444, P. R. China

* Author to whom correspondence should be addressed. Email: genxili@nju.edu.cn

Received: 04 January 2007 / Accepted: 25 January 2007 / Published: 30 January 2007

\begin{abstract}
Camptothecin, an anticancer component from Camptotheca acuminate, may interact with human serum albumin (HSA) at the subdomain IIA (site I), and then convert to its inactive form(carboxylate form). In this paper, the detailed electrochemical behaviors of camptothecin at a pyrolytic graphite electrode is presented. The interaction between camptothecin and HSA is also studied by electrochemical technique. By comparing with bovine serum albumin (BSA), which is highly homologous to HSA, we prove that camptothecin can specifically bind to HSA. Meanwhile, the inhibitory influence of sodium salicylate to this binding is also discussed.
\end{abstract}

Keywords: Camptothecin, human serum albumin, cyclic voltammetry

\section{Introduction}

Camptothecin (Scheme 1), 4-ethyl-4-hydroxy-(S)-1-H-Pyrano [3',4':6,7] indolizino [1,2-6] quinolino-3,14 $(4 \mathrm{H}, 12 \mathrm{H})$-dione, the active compound of Camptotheca acuminate [1], is a well-known antitumor drug for gastric cancer [2], breast cancer [3] and lung cancer [4]. Its primary cellular target is topoisomerase I, and the antitumor activity is attributed to the inhibition of topoisomerase I [5]. As a topoisomerase I "poison", camptothecin can bind both DNA and topoisomerase I to form a stable ternary complex [6,7], which prevents DNA synthesis and induces the death of cells. Meanwhile, it 
has also exhibited some other biological activities, such as anti-HIV [8] and against parasitic trypanosomes and Leishmania [9].

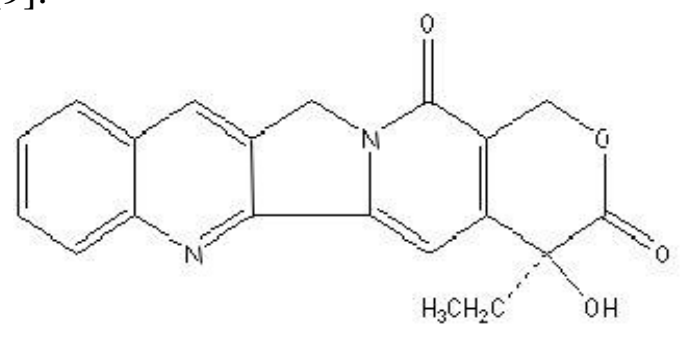

Scheme 1. Chemical structure of camptothecin

There are two forms of camptothecin (the carboxylate form and the lactone form), which exist with different conditions, however, only the lactone one plays the key role in forming the ternary complex [10]. On the other hand, human serum albumin(HSA), the most abundant protein in the serum, can bind to some drug molecules at two binding sites known as site I (subdomain IIA) and site II (subdomain IIIA) [11]. It is reported that in the presence of HSA, camptothecin may interact with the site I of HSA, leading to the conversion of camptothecin from active form to inactive form [12], which evidently reduces the antitumor activities of camptothecin. Thus, the study of the interaction between camptothecin and HSA is highly important for the clinical application of camptothecin. It is also crucial for maintaining the activity of camptothecin to find an effective way of inhibiting this interaction.

In this work, we have used electrochemical method to elucidate the interaction between camptothecin and HSA. By comparing with bovine serum albumin (BSA), we have also proved the existence of the specific binding of camptothecin to HSA. Moreover, the inhibitory influence of sodium salicylate to this specific binding is also discussed.

\section{Results and Discussion}

In order to use electrochemical technique to study the interaction between camptothecin and HSA, we have first studied the electrochemistry of the molecule. Up to now, no voltammetric study of this species has been reported, although the behavior of camptothecin at a hanging mercury drop electrode has been presented [13]. We have found that camptothecin is electroactive at a PG electrode in BrittonRobinson buffer, with the $\mathrm{pH}$ range of 2.0 to 7.0. As shown in Fig. 1, with $4.0 \times 10^{-6} \mathrm{M}$ camptothecin in a pH 4.0, 0.1 M Britton-Robinson buffer solution, a well-defined cathodic peak appears at -0.75 V (vs. SCE) at the PG electrode. Meanwhile, a slight anodic peak is also observed. However, compared with the cathodic peak, the anodic peak is unstable and disappears after several cycles of scanning. In contrast, no voltammetric peak can be obtained at the PG electrode in the absence of camptothecin. Furthermore, the canodic peak rises with the increasing concentration of camptothecin correspondingly. So, the cathodic peak should be attributed to the reduction of camptothecin at the PG electrode. 


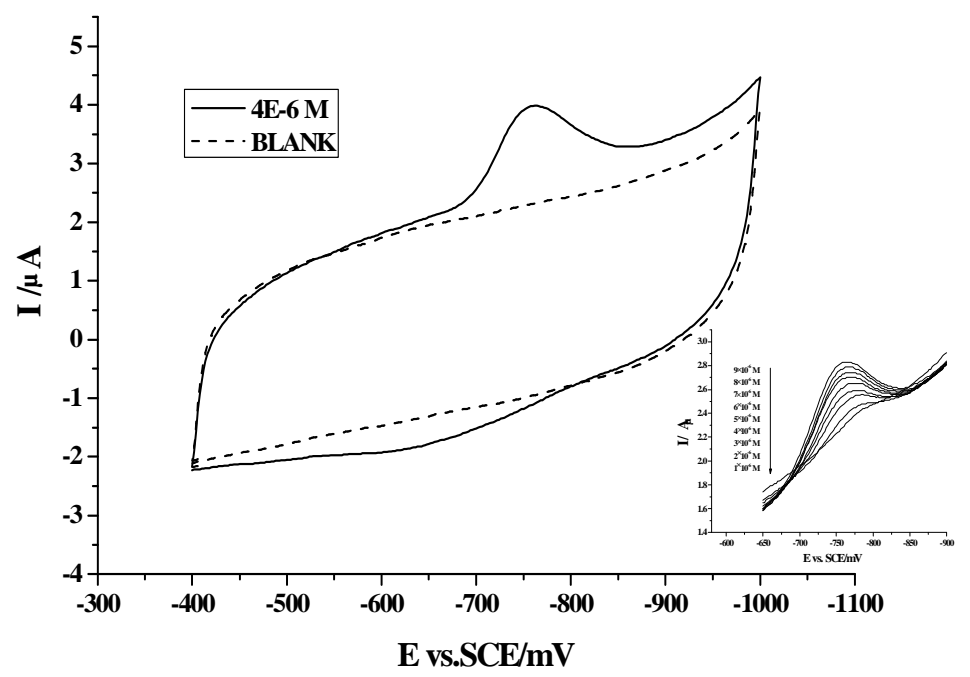

Figure 1. Cyclic voltammogram of camptothecin obtained at a PG electrode in $0.1 \mathrm{M}$ BrittonRobinson buffer solution with $\mathrm{pH} 4.0$ (The first scan). Scan rate: $100 \mathrm{mV} \mathrm{s}^{-1}$. Accumulation time: $60 \mathrm{~s}$. Inset is the cyclic voltammograms of camptothecin solutions with different concentrations.

The effect of scan rate on the voltammetric behavior of camptothecin has been examined (Fig. 2). The cathodic peak current is found to increase linearly with the scan rate from $100 \mathrm{mV} \mathrm{s}^{-1}$ to $700 \mathrm{mV} \mathrm{s}^{-1}$. The linear regression equation is: $y=-0.01814+0.01377 x, \mathrm{r}=0.999$, indicating that the reduction process on the electrode surface is mainly adsorption controlled [14, 15].

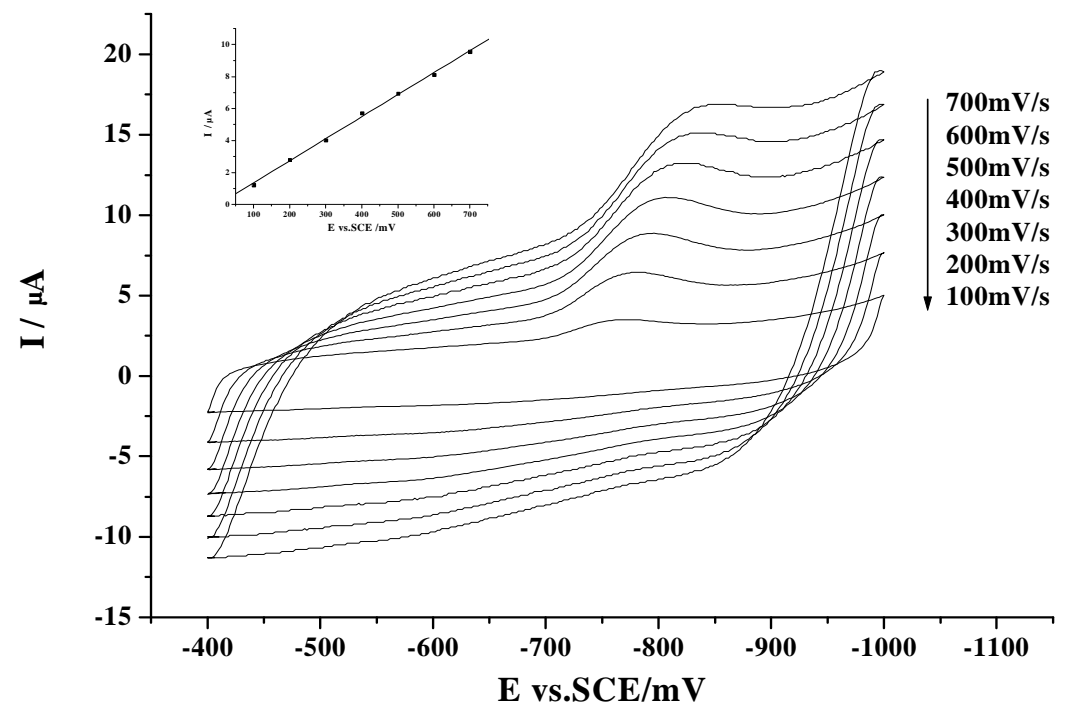

Figure 2. Cyclic voltammograms of camptothecin at scan rates varying from 100 to $700 \mathrm{mV} \mathrm{s}^{-1}$ (from inner to outer), and plot of the cathodic peak current against the scan rate (inset). Others same as in Figure 1.

The $\mathrm{pH}$ value plays an important role in the electrochemical behaviors of camptothecin. Although well-defined voltammograms can be obtained in the $\mathrm{pH}$ range from 2.0 to 7.0 , with the increase of $\mathrm{pH}$ value, the cathodic peak shifts negatively. The cathodic peak potentials are found to be linearly proportional to the $\mathrm{pH}$ value, with a slope $(\Delta E / \Delta p H)$ value of $-71 \mathrm{mV} \mathrm{pH}^{-1}$, which suggests a process 
involving equivalent protons and electrons (Fig. 3A) [16]. The cathodic peak current will also change with the $\mathrm{pH}$ value. As is shown in Fig. 3B, the peak current is the highest at $\mathrm{pH} 5.0$, which is the best for detection purpose. However, it is reported that HSA will preferably bind drugs at pH 4.0 [17]. So, for the studies of the interaction between camptothecin and HSA, we have selected a $\mathrm{pH} 4.0$ buffer solution.

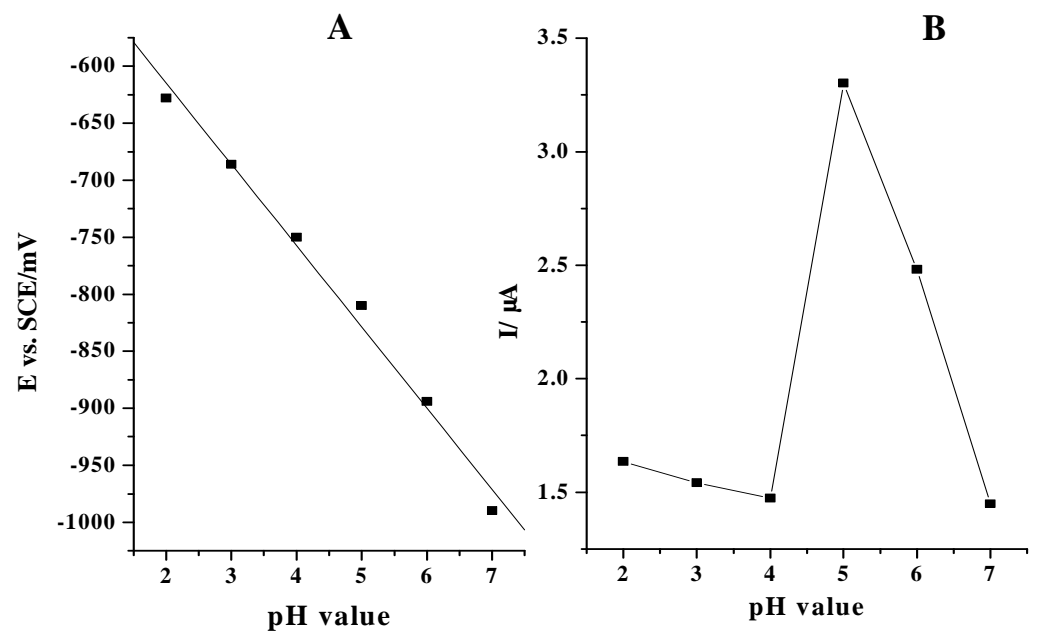

Figure 3. Plot of the cathodic (A) peak potential; (B) peak current against $\mathrm{pH}$ value. Others same as in Figure 1.

Since the electrochemical behavior of camptothecin on the electrode surface is adsorptioncontrolled, it is necessary to investigate the influence of accumulation time. As is shown in Fig. 4, the cathodic peak current increases significantly within the first $60 \mathrm{~s}$, and then slowly and nearly unchanged later on. Thus, the first 60 seconds are chosen as the optimum time period of accumulation.

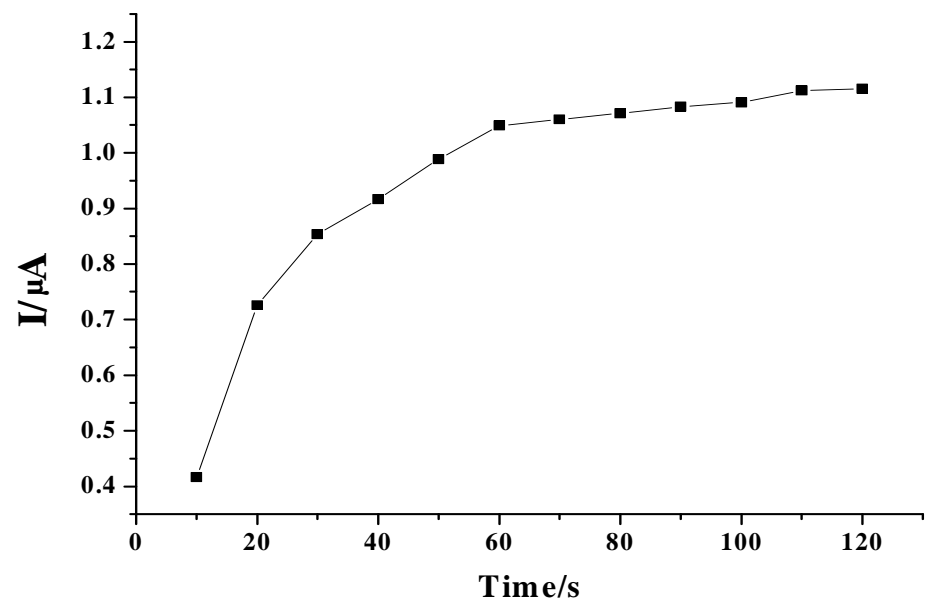

Figure 4. Dependence of the cathodic peak current on the accumulation time.

Others same as in Figure 1.

Further studies reveal that the cathodic peak current increases with the continuous addition of camptothecin and a linear calibration plot $(\mathrm{y}=0.44833+0.19886 \mathrm{x}, \mathrm{r}=0.999)$ can be obtained in the concentration range of $1 \times 10^{-6} \mathrm{M}-1 \times 10^{-5} \mathrm{M}$ (Fig. 5), which suggests that the voltammetric response of 
camptothecin at a PG electrode can be used for the concentration measurements. Although there are several analytical techniques including UV-spectrometry, fluorescence, infrared spectroscopy, and HPLC [18-20] currently available for determining camptothecin, it is still necessary to develop more methods for improving the accuracies of camptothecin measurement [13]. We have thus examined the analytical parameters. The limit of detection (LOD) of this electrochemical method is estimated to be $2 \times 10^{-7} \mathrm{M}$, defined as three times the signal-to-noise ratio, which can be satisfactory compared with the previous report of $8 \times 10^{-7} \mathrm{M}$ [13]. The relative standard deviation (RSD) of five successive determinations at the concentration of $4 \times 10^{-6} \mathrm{M}$ is $5.6 \%$.

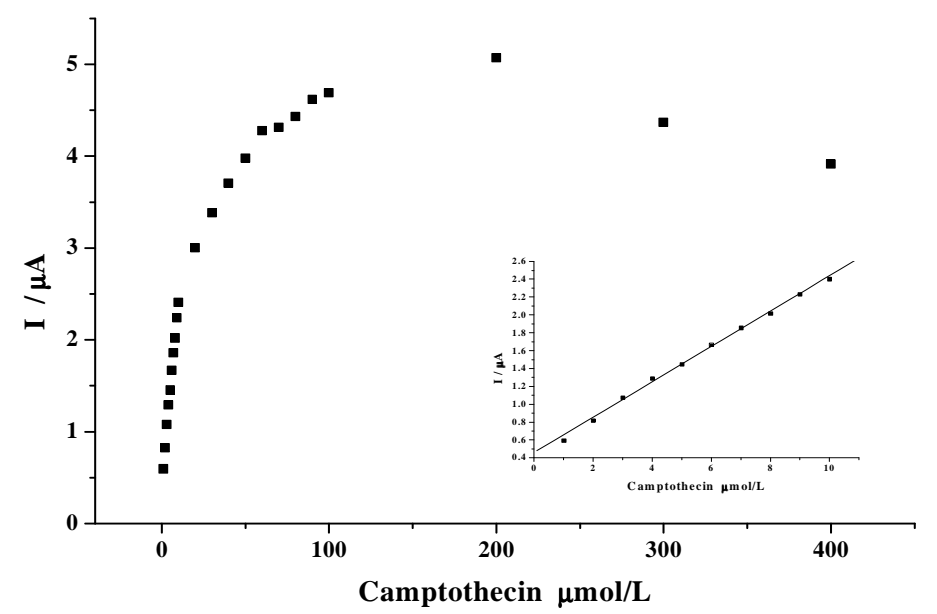

Figure 5. Dependence of the cathodic peak current on the camptothecin concentration. Others same as in Figure 1.

We have then studied the interaction between camptothecin and HSA with electrochemical technique. As shown in Fig. 6, with the addition of HSA into a $0.1 \mathrm{M}$ Britton-Robinson buffer containing $4 \times 10^{-6} \mathrm{M}$ camptothecin, the peak current of camptothecin decreases distinctly, and no appearance of new redox peaks and no shift of the peak potential is observed.

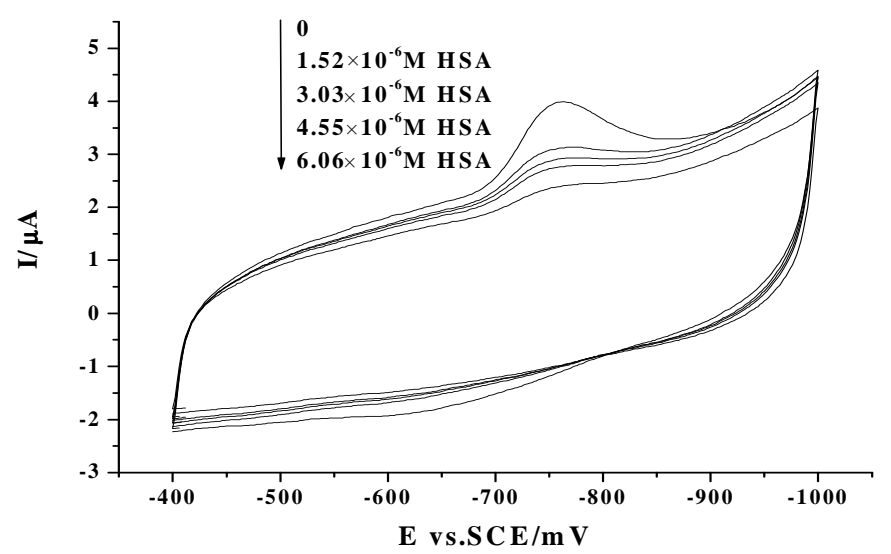

Figure 6. Cyclic voltammograms of $4 \times 10^{-6} \mathrm{M}$ camptothecin in the absence and presence of HSA with different concentrations. Others same as in Figure 1.

There are two possible mechanisms for explanation of this peak decrease. One is that HSA interacts with camptothecin to form a non-electrochemical complex, which blocks the electron transfer between 
camptothecin and electrode. The other is the competitive adsorption of HSA at the PG electrode surface. Since the electrochemical behavior of camptothecin is an adsorptive process, the competitive adsorption of HSA may also reduce the adsorption of camptothecin and cause the decrease of the peak current of camptothecin.

We assume that it is the interaction between HSA and camptothecin and the formation of the nonelectrochemical complex that makes the decrease of peak current of camptothecin. In order to confirm the specific interaction between HSA and camptothecin and the formation of the non-electochemical complex, we have employed BSA as a contrast. HSA and BSA are highly homologous proteins [22], but camptothecin can not bind BSA [12]. If no interaction exists and no complex forms between HSA and camptothecin, the decrease of the peak current of camptothecin caused by HSA and BSA should be almost the same. However, as is shown in Fig. 7 , the decrease caused by BSA is obviously less than HSA. Therefore, the result has not only revealed the existence of the specific binding between camptothecin and HSA, but also suggested that the competitive adsorption of HSA is not the main reason of the decrease of camptothecin peak current.

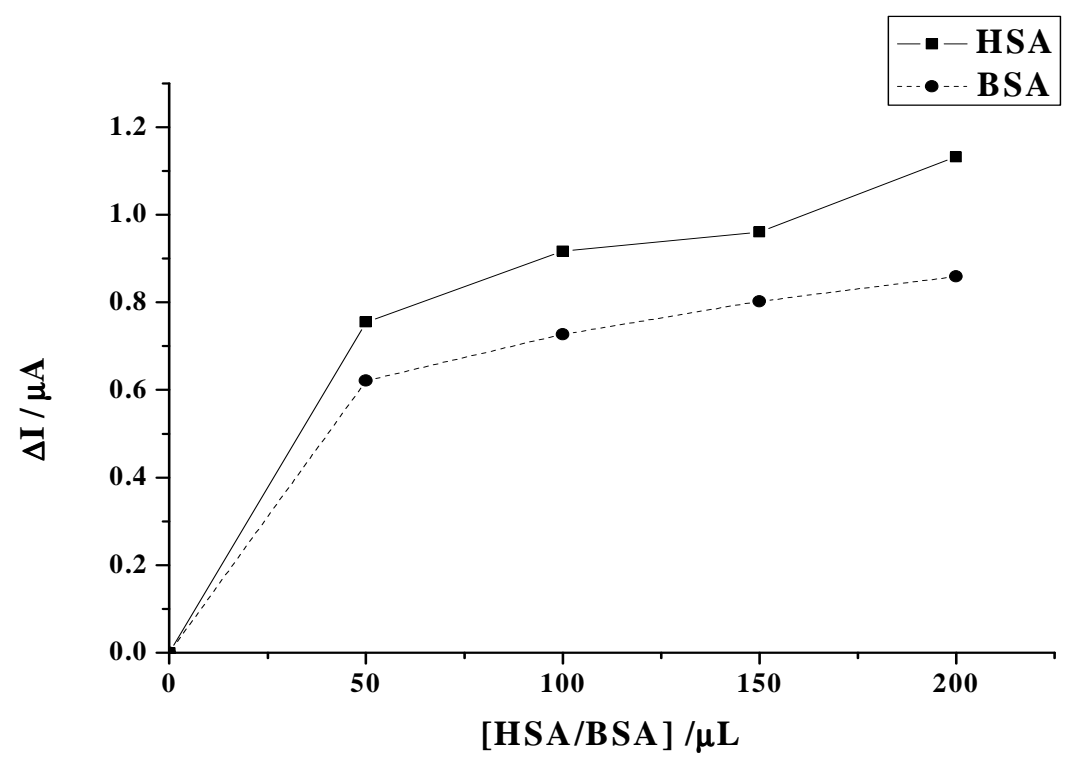

Figure 7. Plot of the decrease of the camptothecin peak current versus the addition of different volume of HSA/BSA in the solutions. HSA/BSA concentration: $10 \mathrm{mg} / \mathrm{ml}\left(1.52 \times 10^{-4} \mathrm{M}\right)$. Others same as in

Figure 1.

Camptothecin has been known to have good antitumor activity. However, we have not used this agent as the treatment for cancers, and we believe that the specific interaction between the molecule and HSA is a major barrier to be overcome. Effort should be made to inhibit the binding of camptothecin to HSA and increase the amount of active camptothecin. It has been known that salicylate can specifically bind the site I of HSA with high affinity [23], so salicylate sodium has been used in this work as a competitive molecule to bind to HSA, which may inhibit the binding of camptothecin with HSA and increase the level of the active camptothecin. As shown in Fig. 8, compared with HSA alone, the decrease of the camptothecin peak current caused by the mixed solution of HSA and salicylate sodium is much less, which indicates that more free camptothecin molecules exist in the presence of salicylate sodium. The reason is that the binding of salicylate to HSA may 
prevent the interaction between camptothecin and HSA. As a result, the free and active camptothecin molecules will increase accordingly.

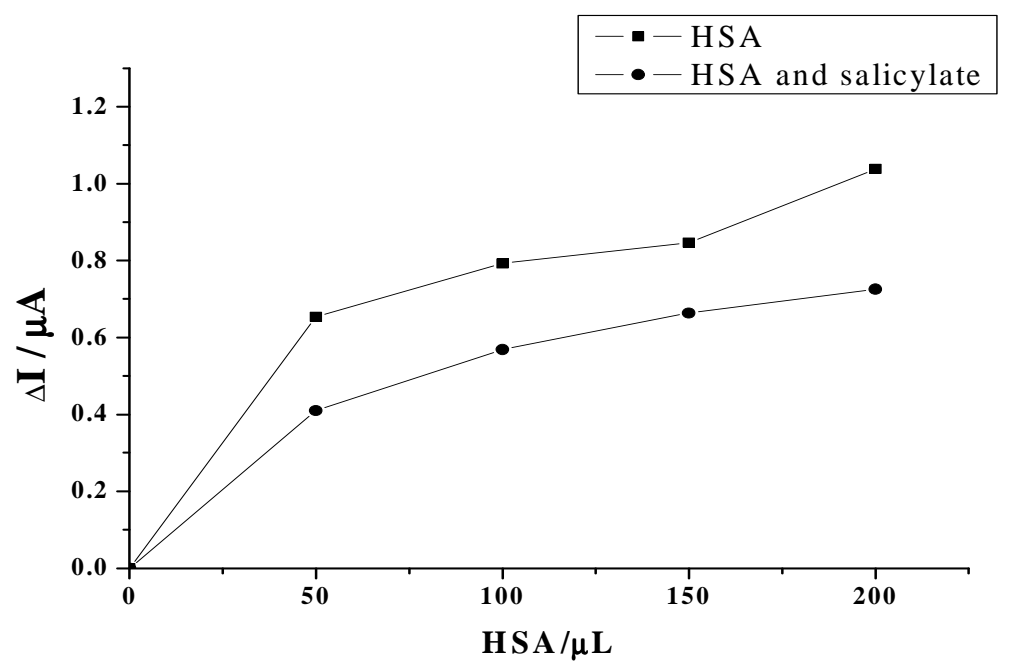

Figure 8. Plot of the decrease of the camptothecin peak current versus the addition of different volume of mixture of HSA and salicylate sodium (The volume ratio of HSA to salicylate is 1:1). The concentration of salicylate sodium: $1.0 \times 10^{-6} \mathrm{M}$. Others same as in Figure 7.

\section{Experimental section}

\section{1 Reagents}

Camptothecin was purchased from Sigma. Stock solutions were prepared by dissolving known amounts of camptothecin in $10 \mathrm{~mL}$ of dimethyl sulphoxide. Salicylate sodium was purchased from Shanghai Shiyi Chemicals Regent Co., Ltd. Stock solutions were prepared by dissolving known amounts of salicylate sodium in $10 \mathrm{~mL}$ of methanol. HSA ( 99\%, molecular mass $69 \mathrm{kDa})$ and BSA ( $\sim 99 \%$, molecular mass $66 \mathrm{kDa}$ ), were purchased from Sigma and used without further purification. Stock solutions were prepared by dissolving known amounts of HSA/BSA in $10 \mathrm{~mL}$ of double-distilled water. Other chemicals were of analytical grade. Double-distilled water, which was purified with a Milli-Q purification system (Branstead, Boston, MA, U.S.A.) to a specific resistance of $>18 \mathrm{M} \Omega \mathrm{cm}$ was used in all experiments, and all solutions were stored in the refrigerator at $4{ }^{\circ} \mathrm{C}$.

\subsection{Preparation of working electrode}

The pyrolytic graphite(PG) rod was purchased from Shanghai Carbon Co. (Shanghai, P.R. China). The substrate PG electrode was prepared by inserting a PG rod in a glass tube and fixing it with epoxy resin. Electrical contact was made by attaching a copper wire to the rod with the help of Wood's alloy (a fusible bismuth-based alloy). For pretreatment, the PG electrode was first polished using rough and fine sand papers. Its surface was then polished to mirror smoothness with alumina (particle size of approx. $0.05 \mu \mathrm{m}$ )/water slurry on silk. Finally, the electrode was thoroughly washed by ultrasonicating in both double-distilled water and ethanol for approx. $5 \mathrm{~min}$. 


\subsection{Apparatus}

Cyclic voltammetry (CV) was performed with a PARC 263A potentiostat/galvanostat (EG\&G; Princeton, NJ, USA), using a three-electrode configuration at $25 \pm 0.5{ }^{\circ} \mathrm{C}$. The reference electrode was a saturated calomel electrode (SCE) and the counter electrode was a platinum electrode. Potentials are reported with respect to SCE unless specially stated. The substrate electrode was a PG electrode $(\mathrm{A}=$ $6.28 \mathrm{~mm}^{2}$ ). All test solutions were thoroughly deoxygenated by bubbling high-purity nitrogen through the solution for at least $10 \mathrm{~min}$. A stream of nitrogen was blown gently across the surface of the solution in order to maintain the solution anaerobic throughout all the experiments.

\section{Acknowledgements}

This work is supported by the National Natural Science Foundation of China (Grant No. 90406005, 20575028) and the Program for New Century Excellent Talents in University, the Chinese Ministry of Education (NCET-04-0452).

\section{References}

1. Marco, E.; Laine, W.; Tardy, C.; Lansiaux, A.; Iwao, M.; Ishibashi, F.; Bailly, C.; Gago, F. Molecular Determinants of Topoisomerase I Poisoning by Lamellarins: Comparison with Camptothecin and Structure-Activity Relationships. J. Med. Chem. 2005, 48, 3796-3807.

2. David A. Litvak; Harry T. Papaconstantinou; B. Mark Evers; Courtney M. Townsend, Jr. Targeting molecular pathways with camptothecin as novel therapy for gastric cancer. Journal of Gastrointestinal Surgery 1999, 3, 618-624.

3. Liu W.M; Zhang R.W. Upregulation of p21 (WAF1/CIP1) in human breast cancer cell lines MCF7 and MDA-MB-468 undergoing apoptosis induced by natural product anticancer drugs 10hydroxycamptothecin and camptothecin through p53-dependent and independent pathways. International Jounal Of Oncology 1998, 12 (4), 793-804.

4. Sanchez-Alcazar JA; Bradbury DA; Brea-Calvo G; Navas P; Knox AJ Camptothecin-induced apoptosis in non-small cell lung cancer is independent of cyclooxygenase expression. Apoptosis 2003, 8 (6), 639-647.

5. Heyong Gao; Xiongwen Zhang; Yi Chen; Hongwu Shen; Tao Pang; Jing Sun; Chenghui Xu; Jian Ding; Chuan Li; Wei Lu Synthesis and antitumor activity of the hexacyclic camptothecin derivatives. Bioorganic \& Medicinal Chemistry Letters 2005, 15, 3233-3236.

6. Hertzberg, R.P.; Busby, R.W.; Caranfa, M.J.; Holden, K.G.; Johnson,R.K.; Hecht, S.M.; Kingsbury, W.D. Irreversible trapping of the DNA-topoisomerase I covalent complex. Affinity labeling of the camptothecin binding site. J. Biol. Chem. 1990, 265, 19287-19295.

7. Ignor Chourpa; Abdel Beljebbar; Ganesh D.Sockalingum; Michel Manfait Structure-activity relation in camptothecin antitumor drugs: why a detailed molecular characterisation of their lactone and carboxylate forms by Raman and SERS spectroscopies? Biochimica et Biophyscia Acta (BBA) -general subjects. 1997, 1334, 349-360. 
8. Priel, E.; Showalter, S. D.; Blair, D.G. Inhibition of human. immnnodeficiency virus (HIV-I) replication by non cytotoxic. doses of camptothecin, a topoisomerase I inhibitor. AIDS Res. Hum. Retrovir. 1991, 7, 65-72.

9. Bodley, A.L.; Shapiro, T.A.; Molecular and cytotoxic effects of camptothecin, a topoisomerase I inhibitor, on trypanosomes and Leishmania. Proc. Natl. Acad. Sci. 1995, 92, 3726-3730.

10. Jaxel, C.; Kohn, K. W.; Wani, M. C.; Wall, M. E. Structure activity study of the actions of camptothecin derivatives on mammalian topoisomerase 1: evidence for a specific receptor site and a relation to antitumor activity. Cancer Res. 1989, 49, 1465-1469.

11. Shane G. Roller; Christine M. Dieckhaus; Webster L. Santos; R. Duane Sofia; Timothy L. Macdonald Interaction between Human Serum Albumin and the Felbamate Metabolites 4Hydroxy-5-phenyl-[1,3]oxazinan-2-one and 2-Phenylpropenal. Chem. Res. Toxicol. 2002, 15, 815824.

12. Fabrice Fleury; Irina Kudelina; Igor Nabiev Interactions of lactone, carboxylate and self-aggregated forms of camptothecin with human and bovine serum albumins. FEBS letter 1997, 406, 151-156.

13. Zheng, J.B; Meng, Z.C; Liu, B.; Zhang, H.F; Investigation on voltammetric behavior of camptothecin and its analytical application. Chinese Journal of Chemistry 2006, 24, 551-556.

14. Ong, E. S.; Apandi, S. N. Determination of berberine and strychnine in medicinal plants and herbal preparations by pressurized liquid extraction with capillary zone electrophoresis. Electrophoresis 2001, 22, 2723-2729.

15. Laviron E. Adsorption, autoinhibition and autocatalysis in polarography and in linear potential sweep voltammetry. J Electroanal Chem 1974, 52, 355-393.

16. Nassar, A.E.F.; Zhang, Z.; Hu, N.F.; Rusling, J.F.; Kumosinski, T.T. Proton-Coupled Electron Transfer from Electrodes to Myoglobin in Ordered Biomembrane-like Films. J.Phys.Chen. B. 1997, 101, 2224-2231.

17. Salman Muzammil; Yogesh Kumar; Saad Tayyab Molten globule-like state of human serum albumin at low pH. Eur. J. Biochem. 1999, 266, 26-32.

18. Li, L. Y.; Zhang, D.Y.; Bai, F.W. Research on Camptothecine (CPT) and its Derivative. Journal of Dalian Nationalities University 2001, 3, 17-22.

19. Liu, W. Z.; Wang, Z.F. Accumulation and Localization of Camptothecin in Young Shoot of Camptotheca acuminate. Acta Photophysiologica Sinica 2004, 30, 405-412.

20. Zhang, Z.X.; Mao, G. X.; Zhang, M.X.; Zhang, W. J. Extracting New Technique for Camptothecine by $\mathrm{NaOH}$ aq. Natural Product Research and Development 2006, 18, 302-303.

21. Yan, X.F.; Wang, Y.; Yu T.; Zhang Y. H.; Yin L.J. Determination of Camptothecin in Leaves of Camptotheca Acuminata Decne. by HPLC. Journal of Instrumental Analysis 2002, 21, 15-17.

22. Hirayama, K.; Akashi, S.; Furuya, M.; Fukuhara, K. I. Rapid confirmation and revision of the primary structure of bovine serum albumin by ESIMS and Frit-FAB LC/MS. Biochem.Biophys. Res. Commun. 1990, 173, 639-646.

23. Inci Özer and Özden Tacal Method Dependence of Apparent Stoichiometry in the Binding of Salicylate Ion to Human Serum Albumin: A Comparison between Equilibrium Dialysis and Fluorescence Titration. Analytical Biochemistry 2001, 294, 1-6.

(C) 2007 by MDPI (http://www.mdpi.org). Reproduction is permitted for noncommercial purposes. 